\title{
Imaging Magnetic Vortices Including Skyrmions by Lorentz TEM and Differential Phase-Contrast Microscopy
}

\author{
Xiuzhen $\mathrm{Yu}^{1 *}$ \\ 1. RIKEN CEMS, Electronic States Microscopy Research Team \\ * Corresponding author: yu_x@riken.jp
}

The nanometric vortex-like topological spin textures, such as magnetic skyrmions and antiskyrmions (magnetic bubbles with two pairs of Bloch lines) have recently attracted enormous attention owing to their topological nature and emergent electromagnetic properties [1]. To confirm such minute complex spin textures and their dynamics in magnets, real-space high-resolution imaging technique, such as Lorentz transmission electron microscopy (TEM) is useful. To quantitatively characterize the topological aspects for the vortex-like spin textures as well as their helicities (in-plane rotating directions of spins), the phase imaging technique, such as differential phase contrast microscopy is effective.

Figure 1a shows the schematic diagram of Lorentz TEM and the defocused Lorentz TEM images for a vortex: Magnetizations perpendicular to the incident electron beam induce a Lorentz force normal to both magnetizations and the electron beam, while the magnetization parallel to the incident electron beam has no magnetic interaction. The Lorentz force induced by perpendicular magnetization deflects the electron beam, resulting in the convergent (bright) and divergent (dark) contrasts in the defocused Lorentz TEM image plane (underfocused or overfocused). The quantitative phase image (Fig. 1b) for magnetic vortices is able to be extracted by analyzing defocused Lorentz TEM images with the transport-of-intensity equation (TIE). On the basis of Maxwell equation, the magnetization map (Fig. 1c) for the vortices can be converted from the phase image [2].

To quantitatively evaluate the phase image of magnetic vortices, in addition to Lorentz TEM combined with TIE analyses, a well-known imaging technique, the differential phase contrast microscopy (DPCM) performed at a scanning TEM (STEM) mode shown in Figs. 2a-b, is powerful. The segment annular detectors can directly sense the in-plane magnetizations along all directions and hence demonstrate the field image (Fig. 2b). Particularly, this technique promises to directly visualize Neel-type skyrmions [3] which are barely imaged at the Lorentz TEM mode, in principle. Figures $2 \mathrm{c}-\mathrm{d}$ demonstrate a helicity image (Fig. 2c) and a field image (Fig. 2d) for a skyrmion lattice (SkL) in a thin plate of chiral-lattice FeGe which agree well with Lorentz TEM results shown in Figs. 1b-c.

In my talk, I will mainly present Lorentz TEM observations of nanometer-scale topological spin textures and their lattice forms, such as meron-antimeron square lattice (sq-ML), hexagonal skyrmion (bubble) lattice (hex-SkL) and their structural transition from the sq-ML into hex-SkL with finely tuning the magnetic field which is applied normally to thin magnets [4-8]. The topological phase transition between a hex-SkL phase and non-topological spin textures, helical or conical structure, has been demonstrated by means of the in-situ Lorentz TEM observations with quenching the thermal equilibrium SkL in a thin helimagnet, FeGe [9]. In addition, the in-situ Lorentz TEM demonstrated skyrmion dynamics, such as the Brownian motion of skyrmions in a chiral-lattice insulator, the collective transformation of sparsely-populated skyrmions to microcrystals of skyrmions, and the current-driven skyrmion motion. Finally, I will show the preliminary data for the Neel-type skyrmions observed by DPCM in multilayers. 
These works were done in collaborations with Profs. Yoshinori Tokura, Naoto Nagaosa, Masashi Kawasaki, Taka-hisa Arima, Maximam Mostovoy, Christopher Marrows, Yusuke Tokunaga, Fumitaka Kagawa and Masahiko Mochizuki, and Drs. Yasujiro Taguchi, Wataru Koshibae, Shinichro Seki, Naoya Kanazawa, Katharina Zeissler, Tomoyuki Yokouchi, Daisuke Morikawa, Masao Nakamura, Kiyou Shibata, and Yoshio Kaneko, Takuro Nagai, Yoshio Matsui, Koji Kimoto, Licong Peng, and Ms. Kiyomi Nakajima.

References:

[1] N. Nagaosa and Y. Tokura, Nat. Nanotechnol. 8, 899 (2013).

[2] K. Ishizuka and B. Allman, J. Electron Microsc. 54, 191 (2005).

[3] J. Sampaio, et al., Nat. Nanotechnol. 8, 839 (2013); K. Zeissler, et al, Sci. Rep. 7, 15125 (2017);

Shawn D. Pollard, et al, Nat. Commun. 8, 1476 (2017).

[4] X. Z. Yu, et al., Nature 465, 901 (2010).

[5] X. Z. Yu, et al., PNAS 109, 8856 (2012).

[6] Y. Tokunaga, et al., Nat. Commun. 6, 7638 (2015).

[7] X. Z. Yu, et al., Nature 564, 95 (2018).

[8] X. Z. Yu, et al., Nat. Phys. 14, 832 (2018).

[9] N. Shibata, et al., Nat. Commun. 8, 15631 (2017); T. Matsumoto, et al., Sci. Adv. 2, e1501280 (2016).

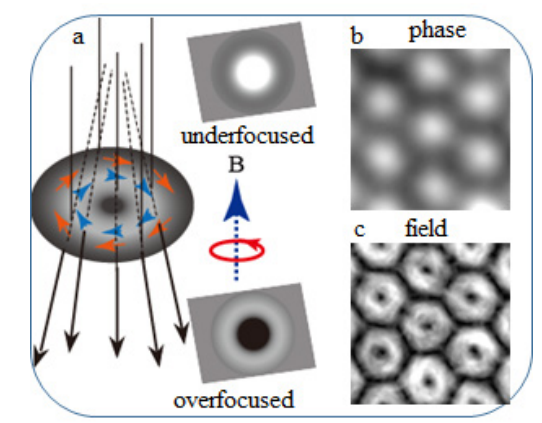

Figure 1 a, Schematic of Lorentz TEM. b and c, Electron phase image and the mapping of magnetic field for a hexagonal lattice of magnetic skyrmions in a thin FeGe observed by Lorentz TEM in combination with transport of intensity equation analyses, respectively.

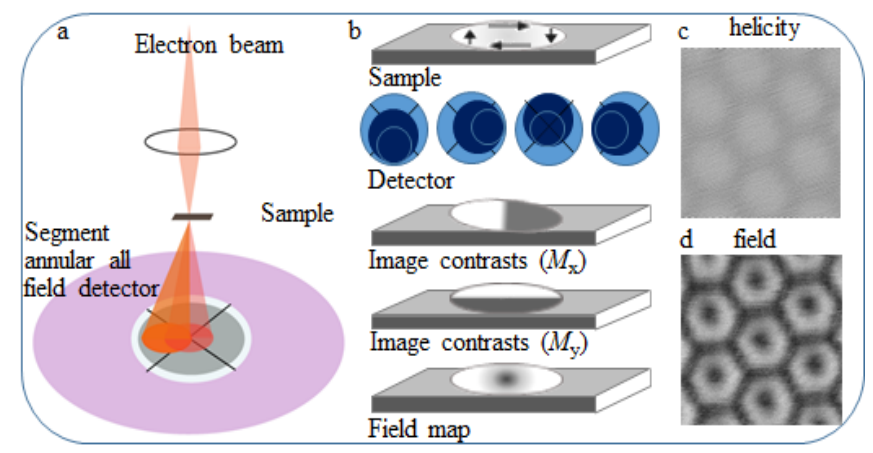

Figure 2 a and b, Schematics of the differential phase contrast microscopy (DPCM) (a) and mapping images of a vortex (b) at the scanning TEM mode, respectively. $\mathrm{c}$ and d, Helicity image and the mapping of magnetic field for a hexagonal lattice of magnetic skyrmions in a thin FeGe observed by DPCM, respectively. 